\title{
Shape Reconstruction for Unsteady Advection-Diffusion Problems by Domain Derivative Method
}

\author{
Wenjing Yan, ${ }^{1,2}$ Jian Su, ${ }^{1,2}$ and Feifei Jing ${ }^{1}$ \\ ${ }^{1}$ School of Mathematics and Statistics, Xian Jiaotong University, Shaanxi 710049, China \\ ${ }^{2}$ Center for Computational Geosciences, Xian Jiaotong University, Shaanxi 710049, China \\ Correspondence should be addressed to Wenjing Yan; wenjingyan@mail.xjtu.edu.cn
}

Received 9 August 2013; Revised 23 January 2014; Accepted 27 January 2014; Published 4 March 2014

Academic Editor: Youyu Wang

Copyright (c) 2014 Wenjing Yan et al. This is an open access article distributed under the Creative Commons Attribution License, which permits unrestricted use, distribution, and reproduction in any medium, provided the original work is properly cited.

\begin{abstract}
This paper is concerned with the numerical simulation for shape reconstruction of the unsteady advection-diffusion problems. The continuous dependence of the solution on variations of the boundary is established, and the explicit representation of domain derivative of corresponding equations is derived. This allows the investigation of iterative method for the ill-posed problem. By the parametric method, a regularized Gauss-Newton scheme is employed to the shape inverse problem. Numerical examples indicate that the proposed algorithm is feasible and effective for the practical purpose.
\end{abstract}

\section{Introduction}

The advection-diffusion problem is important to many branches of science and engineering. Many physical and chemical phenomena, such as the diffusion of polluted substances in water and air, the diffusion of heat and salinity in the ocean, and even economics and financial forecasting, can be described as advection-diffusion problems.

For the shape reconstruction problems by the domain derivative method, many people are contributed to it. Hettlich solved the inverse obstacle scattering problem for sound soft and sound hard obstacles $[1,2]$ and discussed a discontinuity in a conductivity from a single boundary measurement [3]. Kress and Rundell considered an inverse conduction scattering problem for shape and impedance in [4]. Chapko et al. dealt with the inverse boundary problem for the time-dependent heat equation only in the case of perfectly conducting and insulating inclusions $[5,6]$. Harbrecht and Tausch considered the numerical solution of a shape identification problem for the heat equation $[7,8]$. Yan and $\mathrm{Ma}$ discussed a shape reconstruction problem for heat conduction with mixed condition [9] and recovered the shape of a solid immersed in the incompressible fluid driven by the Stokes flow [10].
This paper is organized into four parts. In Section 2, we briefly introduce the shape reconstruction problem of the two-dimensional advection-diffusion equations for the transport of a contaminant in the surface water. In Section 3, we describe the domain perturbation method which is used for the characterization of the deformation of the shapes and derive the explicit representation of the derivative of solution with respect to the boundary, that is, the socalled "domain derivative." This representation is important, because it is the key to deriving many properties of the domain derivative method for numerical analysis. Section 4 is devoted to the regularized Gauss-Newton scheme applied to the numerical shape inverse problem. The performance of the numerical method is discussed and illustrated by numerical examples.

\section{Shape Reconstruction Problem}

In this paper, we pay our attention on reconstructing the shape of a bounded and smooth domain from observed information. Let $S$ be a domain contained in a bounded domain $D \in \mathbb{R}^{2}$. The boundaries of $D$ and $S$ are assumed to be $C^{2}$ smooth and defined by $\Gamma_{1}$ and $\Gamma_{2}$, respectively. We denote 
$\Omega:=D \backslash \bar{S}$ and consider the two-dimensional advectiondiffusion equations for the transport of a contaminant in the surface water with an initial condition

$$
\begin{gathered}
\frac{\partial u}{\partial t}+\mathbf{b} \cdot \nabla u-\operatorname{div}(a \nabla u)=f \quad \mathbf{x} \in \Omega, t \in(0, T], \\
u(\mathbf{x}, t)=0 \quad \mathbf{x} \in \Gamma_{1}, t \in(0, T], \\
u(\mathbf{x}, t)=0 \quad \mathbf{x} \in \Gamma_{2}, t \in(0, T], \\
u(\mathbf{x}, 0)=u_{0} \quad \mathbf{x} \in \Omega,
\end{gathered}
$$

where $t$ is time, $\mathbf{x}=(x, y)$ represents the space coordinate, $a$ is diffusion coefficient, $u(\mathbf{x}, t)$ is concentration of the contaminant, $\mathbf{b}$ denotes velocity of water flow, and $f$ accounts for external sources and sinks, respectively. Notice that $a$ and $\mathbf{b}$ are considered to be positive constants quantifying the diffusion and advection processes. For a given domain $D$, it is well known that this initial boundary value problem has a unique solution [11].

The purpose of this paper is to investigate the feasibility of recovering the unknown boundary $\Gamma_{2}$ from the measured (observed) data on the exterior boundary. We define the operator $F$ on the admissible set $X$ by $F\left(\Gamma_{2}\right)=P$, where $P$ is the measured (observed) data and may represent a given objective related to specific characteristic features of the diffusion and advection processes. The inverse problem is both ill-posed and nonlinear.

First of all, we introduce the following functional spaces which will be used throughout this paper. Let $L^{2}(\Omega)$ be the space of square integrable real-valued functions on $\Omega$ with the usual norm. The space $H^{k}(\Omega)$, where $k=1,2 \ldots, n$ denotes the standard Sobolev space on $\Omega$ (see [12]), that is, the space of functions with generalized derivatives of order up to $k$ in $L^{2}(\Omega)$. In addition, $H_{0}^{1}(\Omega):=\left\{v \in H^{1}(\Omega),\left.v\right|_{\partial \Omega}=0\right\}$.

Multiplying the advection-diffusion equations (1) by a test function $v \in H_{0}^{1}(\Omega)$, we easily derive the variational formulation as follows.

Find $u \in H_{0}^{1}(\Omega)$, such that

$$
\int_{\Omega} \frac{\partial u}{\partial t} v \mathrm{~d} x+\int_{\Omega} \mathbf{b} \cdot \nabla u v \mathrm{~d} x+\int_{\Omega} a \nabla u \cdot \nabla v \mathrm{~d} x=\int_{\Omega} f v \mathrm{~d} x,
$$

for all $v \in H_{0}^{1}(\Omega)$. The above identity has to be intended in the sense of distribution in $(0, T]$ and can be expressed in the finite element method framework as

$$
t(u, v)+a(u, v)+c(u, v)=(f, v) \quad \forall v \in H_{0}^{1}(\Omega),
$$

where the forms are given by

$$
\begin{array}{cc}
a(u, v)=\int_{\Omega} a \nabla u \cdot \nabla v \mathrm{~d} x, & c(u, v)=\int_{\Omega} \mathbf{b} \cdot \nabla u v \mathrm{~d} x, \\
t(u, v)=\int_{\Omega} \frac{\partial u}{\partial t} v \mathrm{~d} x, & (f, v)=\int_{\Omega} f v \mathrm{~d} x .
\end{array}
$$

\section{Domain Derivative Method}

This section is devoted to deriving the domain derivative of the solution of the advection-diffusion equations.
A derivative of operator $F$ at boundary $\Gamma$ can be defined as follows [13]. For any real vector field $\mathbf{a} \in C^{2}\left(\Gamma ; \mathbb{R}^{2}\right)$, we denote by $\Gamma_{a}$ the set

$$
\Gamma_{a}=\{\mathbf{x}+\mathbf{a}(\mathbf{x}), \mathbf{x} \in \Gamma\},
$$

where $\|\mathbf{a}\|_{\infty}:=\max _{\mathbf{x} \in \Gamma}|\mathbf{a}|$ is small enough. Now we define the domain derivative of $F$ at boundary $\Gamma$ in the direction a by

$$
F^{\prime}(\Gamma ; \mathbf{a}):=\lim _{\epsilon \rightarrow 0} \frac{1}{\epsilon}\left[F\left(\Gamma_{\epsilon a}\right)-F(\Gamma)\right],
$$

where the limit should exist uniformly.

Similarly, if the vector field $\mathbf{h} \in C^{2}\left(\Gamma_{2}\right)$ is sufficiently small, a perturbation of the boundary $\Gamma_{2}$ can be specified by

$$
\Gamma_{2}^{h}=\left\{\mathbf{x}+\mathbf{h}(\mathbf{x}), \mathbf{x} \in \Gamma_{2}\right\},
$$

which is a $C^{2}$ boundary of a perturbed domain $\Omega_{h}$. We choose an extension of $\mathbf{h} \in C^{2}(\Omega)$ with $\|\mathbf{h}\|_{C^{2}(\Omega)} \leq c\|\mathbf{h}\|_{C^{2}\left(\Gamma_{2}\right)}, c>0$, which vanishes in the exterior of a neighborhood of $\Gamma_{2}$ and define the diffeomorphism $\varphi(\mathbf{x})=\mathbf{x}+\mathbf{h}(\mathbf{x})$ in $\Omega$. If the inverse function of $\varphi$ is denoted by $\psi, J_{\varphi}$ and $J_{\psi}$ are Jacobian matrices.

Let us consider $u_{h} \in H_{0}^{1}\left(\Omega_{h}\right)$ to be the solution of perturbed problem, for a variation $\mathbf{h}$ given by the solution of

$$
\begin{aligned}
& \int_{\Omega_{h}} \frac{\partial u_{h}}{\partial t} v_{h} \mathrm{~d} x_{h}+\int_{\Omega_{h}} \mathbf{b}_{h} \cdot \nabla u_{h} v_{h} \mathrm{~d} x_{h} \\
& \quad+\int_{\Omega_{h}} a_{h} \nabla u_{h} \cdot \nabla v_{h} \mathrm{~d} x_{h}=\int_{\Omega_{h}} f_{h} v_{h} \mathrm{~d} x_{h},
\end{aligned}
$$

for all $v_{h} \in H_{0}^{1}\left(\Omega_{h}\right)$ with $a_{h}, \mathbf{b}_{h}$, and $f_{h}$ defined as $a, \mathbf{b}$, and $f$ replacing $\Omega$ by $\Omega_{h}$. Changing the spatial variables by the diffeomorphism $\varphi$ leads to

$$
\begin{gathered}
\int_{\Omega} \frac{\partial \tilde{u}}{\partial t} v \operatorname{det}\left(J_{\varphi}\right) \mathrm{d} x+\int_{\Omega} \tilde{\mathbf{b}} \cdot \nabla \tilde{u} v J_{\psi} \operatorname{det}\left(J_{\varphi}\right) \mathrm{d} x \\
\quad+\int_{\Omega} \tilde{a} \nabla \tilde{u}\left(J_{\psi}\left(J_{\psi}\right)^{T} \operatorname{det}\left(J_{\varphi}\right)\right) \cdot \nabla v \mathrm{~d} x \\
=\int_{\Omega} \tilde{f} v \operatorname{det}\left(J_{\varphi}\right) \mathrm{d} x,
\end{gathered}
$$

for all $v \in H_{0}^{1}(\Omega)$, where $\tilde{a}=a_{h} \circ \psi, \widetilde{\mathbf{b}}=\mathbf{b}_{\mathbf{h}} \circ \psi$, and $\tilde{f}=f_{h} \circ \psi$. The Jacobian matrix of $\mathbf{h}$ is abbreviated by $J_{h}$. From $J_{\varphi}=$ $I+J_{h}$ and $J_{\psi}=J_{\varphi}^{-1} \circ \psi=I-J_{h}+O\left(\|\mathbf{h}\|_{C^{2}(\Omega)}^{2}\right)$, the following first-order expansions hold

$$
\begin{gathered}
\left\|\tilde{a} J_{\psi}\left(J_{\psi}\right)^{T} \operatorname{det}\left(J_{\varphi}\right)-a I+a\left(J_{h}+J_{h}^{T}\right)-\operatorname{div}(a \mathbf{h}) I\right\|_{\infty} \\
=O\left(\|\mathbf{h}\|_{C^{2}(\Omega)}^{2}\right), \\
\left\|\tilde{\mathbf{b}} \cdot J_{\psi} \operatorname{det}\left(J_{\varphi}\right)-\mathbf{b}+\mathbf{b} \cdot J_{h}-\operatorname{div}(\mathbf{b} \cdot \mathbf{h}) I\right\|_{\infty} \\
=O\left(\|\mathbf{h}\|_{C^{2}(\Omega)}^{2}\right), \\
\left\|\tilde{f} \operatorname{det}\left(J_{\varphi}\right)-f-\operatorname{div}(f \mathbf{h})\right\|_{\infty}=O\left(\|\mathbf{h}\|_{C^{2}(\Omega)}^{2}\right) .
\end{gathered}
$$

Furthermore, we can prove the following important theorem which is the main theoretical result of the paper. 
Theorem 1. Assume that $S$ is a domain with boundary $\Gamma_{2}$ in the class $C^{2}$, and $u \in H_{0}^{1}(\Omega)$ denotes the solution of (1). Then $u$ is differentiable at $\Gamma_{2}$ in the sense that there exists $u^{*}$ depending on $\mathbf{h}$, such that

$$
\lim _{\mathbf{h} \rightarrow 0} \frac{1}{\|h\|_{C^{2}}}\left\|\tilde{u}-u-u^{*}\right\|_{1}=0
$$

Moreover, the domain derivative $u^{\prime}$ satisfies the initial boundary value problem

$$
\begin{gathered}
\frac{\partial u^{\prime}}{\partial t}+\mathbf{b} \cdot \nabla u^{\prime}-\operatorname{div}\left(a \nabla u^{\prime}\right)=0 \quad \mathbf{x} \in \Omega, t \in(0, T] \\
u^{\prime}=0 \quad \mathbf{x} \in \Gamma_{1}, t \in(0, T] \\
u^{\prime}=-h_{n} \frac{\partial u}{\partial \mathbf{n}} \quad \mathbf{x} \in \Gamma_{2}, t \in(0, T] \\
u^{\prime}(\mathbf{x}, 0)=0 \quad \mathbf{x} \in \Omega
\end{gathered}
$$

where $h_{n}=\mathbf{h} \cdot \mathbf{n}$ is the normal component of the vector field $\mathbf{h}$.

Proof. Firstly, we will establish the continuous dependence of the solution $u$ on variations of the boundary $\Gamma_{2}$. We consider the difference $\tilde{u}-u$, and the variational equation yields

$$
\begin{aligned}
t(\tilde{u}- & u, v)+a(\tilde{u}-u, v)+c(\widetilde{u}-u, v) \\
= & t(\widetilde{u}, v)-t(A \widetilde{u}, v)+t(A \widetilde{u}, v)-t(u, v) \\
& +a(\widetilde{u}, v)-a(B \tilde{u}, v)+a(B \tilde{u}, v)-a(u, v) \\
& +c(\widetilde{u}, v)-c(C \tilde{u}, v)+c(C \tilde{u}, v)-c(u, v) \\
= & t((I-A) \tilde{u}, v)+a((I-B) \tilde{u}, v) \\
& +c((I-C) \tilde{u}, v)+\int_{\Omega} \tilde{f} v A \mathrm{~d} x-\int_{\Omega} f v \mathrm{~d} x,
\end{aligned}
$$

where the notations $A:=\operatorname{det}\left(J_{\varphi}\right), B:=J_{\psi}\left(J_{\psi}\right)^{T} \operatorname{det}\left(J_{\varphi}\right)$, and $C:=J_{\psi} \operatorname{det}\left(J_{\varphi}\right)$.

From the first-order approximations (10) in $\Omega$ and taking $v=\widetilde{u}-u$, the perturbation argument shows continuity

$$
\|\tilde{u}-u\|_{1} \longrightarrow 0, \quad \text { as }\|\mathbf{h}\|_{C^{2}(\Omega)} \longrightarrow 0 .
$$

Secondly, in order to prove the differentiability of the solution $u$ with respect to the boundary $\Gamma_{2}$, we define $u^{*}=$ $u^{\prime}+\mathbf{h} \cdot \nabla u$ with the extension of $\mathbf{h}$ in $\Omega$. Notice that in shape optimization, $u^{*}$ is always called the material derivative (see [14]). Moreover, $u^{*}$ is the unique solution of

$$
\begin{aligned}
& t\left(u^{*}, v\right)+a\left(u^{*}, v\right)+c\left(u^{*}, v\right) \\
& =\int_{\Omega} \frac{\partial u}{\partial t} \operatorname{div} \mathbf{h d} x \\
& \quad+\int_{\Omega} \nabla u \cdot\left(a\left(J_{h}+J_{h}^{T}\right)-\operatorname{div}(a \mathbf{h}) I\right) \nabla v \mathrm{~d} x \\
& \quad+\int_{\Omega}(\mathbf{h} \cdot \nabla u) \operatorname{div} \mathbf{h} v \mathrm{~d} x+\int_{\Omega} \operatorname{div}(f \mathbf{h}) v \mathrm{~d} x,
\end{aligned}
$$

for all $v \in H_{0}^{1}(\Omega)$. According to the definitions of forms $a(\cdot, \cdot)$, $b(\cdot, \cdot)$, and $t(\cdot, \cdot)$, the identity follows

$$
\begin{aligned}
& t\left(\tilde{u}-u-u^{*}, v\right)+a\left(\widetilde{u}-u-u^{*}, v\right)+c\left(\widetilde{u}-u-u^{*}, v\right) \\
& =t(\widetilde{u}-u, v)-t\left(u^{*}, v\right)+a(\widetilde{u}-u, v)-a\left(u^{*}, v\right) \\
& \quad+c(\widetilde{u}-u, v)-c\left(u^{*}, v\right) .
\end{aligned}
$$

Plugging (13) and (15) into the above equation, we obtain

$$
\begin{gathered}
t\left(\tilde{u}-u-u^{*}, v\right)+a\left(\tilde{u}-u-u^{*}, v\right)+c\left(\tilde{u}-u-u^{*}, v\right) \\
=\int_{\Omega} \frac{\partial \tilde{u}}{\partial t} v\left(I-\operatorname{det}\left(J_{\varphi}\right)\right) \mathrm{d} x-\int_{\Omega} \frac{\partial u}{\partial t} \operatorname{div} \mathbf{h} \mathrm{d} x \\
\quad+\int_{\Omega} \nabla \widetilde{u}\left(a I-\widetilde{a} J_{\psi}\left(J_{\psi}\right)^{T} \operatorname{det}\left(J_{\varphi}\right)\right) \cdot \nabla v \mathrm{~d} x \\
\quad-\int_{\Omega} \nabla u \cdot\left(a\left(J_{h}+J_{h}^{T}\right)-\operatorname{div}(a \mathbf{h}) I\right) \nabla v \mathrm{~d} x \\
\quad+\int_{\Omega} \nabla u \cdot\left(\mathbf{b}-\widetilde{\mathbf{b}} J_{\psi} \operatorname{det}\left(J_{\varphi}\right)\right) v \mathrm{~d} x \\
\quad-\int_{\Omega} \operatorname{div}(\mathbf{b} \cdot \mathbf{h}) v \mathrm{~d} x+\int_{\Omega}\left(\tilde{f} \operatorname{det}\left(J_{\varphi}\right)-f\right) v \mathrm{~d} x .
\end{gathered}
$$

Taking $v=\tilde{u}-u-u^{*}$ and applying the first-order expansions again and the continuity of $\widetilde{u}_{h}$, we have

$$
\frac{1}{\|\mathbf{h}\|_{C^{2}}}\left\|\tilde{u}-u-u^{*}\right\|_{1} \longrightarrow 0, \quad \text { as }\|\mathbf{h}\|_{C^{2}} \longrightarrow 0
$$

Finally, we have to show that $u^{*}$ can be split into $\mathbf{h} \cdot \nabla u$ and $u^{\prime}$. By the chain rule, the formula

$$
\begin{aligned}
\nabla u \cdot[ & \left.a\left(J_{h}+J_{h}^{T}\right)-\operatorname{div}(a \mathbf{h}) I\right] \nabla v \\
= & -(\mathbf{h} \cdot \nabla u) \operatorname{div}(a \nabla v)-(\mathbf{h} \cdot \nabla v) \operatorname{div}(a \nabla u) \\
& +\operatorname{div}[a(\mathbf{h} \cdot \nabla u) \nabla v+(\mathbf{h} \cdot \nabla v)(a \nabla u)-(a \nabla u \cdot \nabla v) \mathbf{h}]
\end{aligned}
$$

holds, if $a, u$, and $v$ are sufficiently smooth. Applying the Gauss formula, the equation yields

$$
\begin{aligned}
& \int_{\Omega} \operatorname{div} \mathbf{w d} x \\
& \quad=\int_{\Omega} \operatorname{div}[(\mathbf{h} \cdot \nabla u) \nabla v+(\mathbf{h} \cdot \nabla v) \nabla u-(\nabla u \cdot \nabla v) \mathbf{h}] \mathrm{d} x \\
& =\int_{\partial \Omega}[(\mathbf{h} \cdot \nabla u) \nabla v+(\mathbf{h} \cdot \nabla v) \nabla u-(\nabla u \cdot \nabla v) \mathbf{h}] \cdot \mathbf{n d} s \\
& =\int_{\partial \Omega}(\mathbf{h} \cdot \nabla u) \nabla v \cdot \mathbf{n d} s,
\end{aligned}
$$

with the abbreviation $\mathbf{w}=(\mathbf{h} \cdot \nabla u) \nabla v+(\mathbf{h} \cdot \nabla v) \nabla u-(\nabla u \cdot \nabla v) \mathbf{h}$. 
From Green formula and the above equation, we have

$$
\begin{aligned}
& a(\mathbf{h} \cdot \nabla u, v) \\
& =-\int_{\Omega} a(\mathbf{h} \cdot \nabla u) \Delta v \mathrm{~d} x+\int_{\partial \Omega} a(\mathbf{h} \cdot \nabla u) \nabla v \cdot \mathbf{n d} s \\
& =\int_{\Omega} a(\operatorname{div} \mathbf{w}-(\mathbf{h} \cdot \nabla u) \Delta v-(\mathbf{h} \cdot \nabla v) \Delta u) \mathrm{d} x \\
& \quad+\int_{\Omega} a(\mathbf{h} \cdot \nabla v) \Delta u \mathrm{~d} x .
\end{aligned}
$$

Notice that $u$ satisfies the advection-diffusion equations (1) and applies the geometrical decompositions formulae [13], and we can obtain

$$
\begin{aligned}
& a(\mathbf{h} \cdot \nabla u, v) \\
& =\int_{\Omega} a(\operatorname{div} \mathbf{w}-(\mathbf{h} \cdot \nabla u) \Delta v-(\mathbf{h} \cdot \nabla v) \Delta u) \mathrm{d} x \\
& \quad+\int_{\Omega} \operatorname{div} \mathbf{h} v\left(f-\mathbf{b} \cdot \nabla u-\frac{\partial u}{\partial t}\right) \mathrm{d} x \\
& \quad+\int_{\Omega} v\left(\mathbf{h} \cdot \nabla\left(f-\mathbf{b} \cdot \nabla u-\frac{\partial u}{\partial t}\right)\right) \mathrm{d} x
\end{aligned}
$$

Similarly, the following equation holds

$$
\begin{aligned}
c(\mathbf{h} \cdot \nabla u, v) & =\int_{\Omega} \mathbf{b} \cdot \nabla(\mathbf{h} \cdot \nabla u) v \mathrm{~d} x \\
& =\int_{\Omega} \mathbf{b} \cdot(\operatorname{div} \mathbf{h} \cdot \nabla u+\mathbf{h} \Delta u) v \mathrm{~d} x .
\end{aligned}
$$

We subtract (22) and (23) from (15); hence the difference is equal to

$$
\begin{aligned}
& t\left(u^{*}-\mathbf{h} \cdot \nabla u, v\right)+a\left(u^{*}-\mathbf{h} \cdot \nabla u, v\right)+b\left(u^{*}-\mathbf{h} \cdot \nabla u, v\right) \\
& =\int_{\Omega} \frac{\partial u}{\partial t} \operatorname{div} \mathbf{h} \mathrm{d} x \\
& \quad+\int_{\Omega} \nabla u \cdot\left(a\left(J_{h}+J_{h}^{T}\right)-\operatorname{div}(a \mathbf{h}) I\right) \nabla v \mathrm{~d} x \\
& \quad+\int_{\Omega}(\mathbf{h} \cdot \nabla u) \operatorname{div} \mathbf{h} v \mathrm{~d} x+\int_{\Omega} \operatorname{div}(f \mathbf{h}) v \mathrm{~d} x \\
& \quad-\int_{\Omega} a(\operatorname{div} \mathbf{w}-(\mathbf{h} \cdot \nabla u) \Delta v-(\mathbf{h} \cdot \nabla v) \Delta u) \mathrm{d} x \\
& \quad-\int_{\Omega}(\mathbf{h} \cdot \nabla v)\left(f-\mathbf{b} \cdot \nabla u-\frac{\partial u}{\partial t}\right) \mathrm{d} x \\
& \quad-\int_{\Omega} \mathbf{b} \cdot(\operatorname{div} \mathbf{h} \cdot \nabla u+\mathbf{h} \Delta u) v \mathrm{~d} x=0 .
\end{aligned}
$$

From $u^{*}=\mathbf{h} \cdot \nabla u+u^{\prime}$, the last equation implies $t\left(u^{\prime}, v\right)+$ $a\left(u^{\prime}, v\right)+b\left(u^{\prime}, v\right)=0$; namely,

$$
\frac{\partial u^{\prime}}{\partial t}+\mathbf{b} \cdot \nabla u^{\prime}-a \Delta u^{\prime}=0, \quad \mathbf{x} \in \Omega .
$$

Note that $u^{*}$ vanishes on the boundary $\Gamma_{2},\left.u\right|_{\Gamma_{2}}=0$ implies $\left.\nabla_{\tau} u\right|_{\Gamma_{2}}=0$, and we derive the boundary condition on $\Gamma_{2}$,

$$
u^{\prime}=u^{*}-\mathbf{h} \cdot \nabla u=-\left(\mathbf{h}_{\tau} \cdot \nabla_{\tau} u+\mathbf{h} \cdot \frac{\partial u}{\partial \mathbf{n}} \mathbf{n}\right)=-h_{n} \frac{\partial u}{\partial \mathbf{n}} .
$$

Therefore, $u^{\prime}$ satisfies the initial boundary value problem (12). This ends the proof.

\section{Numerical Algorithm and Examples}

In this section, we will present a regularized Gauss-Newton algorithm and numerical examples in two dimensions to verify that our methods could be very useful and efficient for the shape reconstruction problem of unsteady advectiondiffusion equations.

From the numerous methods which have been developed for the solution of inverse boundary value problems of this type, we note two groups of approaches, namely, regularized Gauss-Newton iterations and decomposition methods. We choose the regularized Gauss-Newton method in this paper.

Newton method is based on the observed information. We define an operator $F$ on set $X$ of admissible boundaries by

$$
F\left(\Gamma_{2}\right)=P
$$

where $P$ is the measured (observed) data [14], $X:=\{\varphi \in$ $\left.C^{2}\left(\Gamma_{2}\right), 0<\beta \leq\|\varphi\|_{C^{2}} \leq \gamma\right\}$, and $\varphi$ is the parametrized form of boundary $\Gamma_{2}$.

However, since the linearized version of (27) inherits the ill-posedness, the Newton iterations need to be regularized. This approach has the advantages that, in principle, it is conceptually simple and that it leads to highly accurate reconstructions. But, as disadvantages, we notice that the numerical implementation requires the forward solution of the problem (1) in each step of the Newton iteration and reasonable a priori information for the initial approximation.

4.1. Parametric Technique of Boundary. A numerical implementation requires a parametrization of the boundary. Here we apply the parametric representations

$$
\Gamma_{k}:=\left\{X_{k}(\theta)=\left(x_{k, 1}(\theta), x_{k, 2}(\theta)\right), 0 \leqslant \theta<2 \pi\right\} \quad k=1,2,
$$

where $X_{k}: \mathbb{R} \rightarrow \mathbb{R}^{2}$ is twice differentiable and $2 \pi$ periodic with $\left|X_{k}(\theta)\right|>0$ for all $\theta$. Further we assume that the orientation of the parametrization $x_{1}$ is clockwise and the parametrization $x_{2}$ is counter clockwise. In addition, we assume that $\Gamma_{2}$ is starlike with respect to the origin; that is,

$$
X_{\alpha}(\theta)=r_{\alpha}(\theta)\left(\begin{array}{c}
\cos \theta \\
\sin \theta
\end{array}\right), \quad 0 \leqslant \theta<2 \pi,
$$

where

$$
r_{\alpha}(\theta)=\alpha_{0}+\sum_{j=1}^{M}\left(\alpha_{j} \cos j \theta+\alpha_{j+M} \sin j \theta\right)
$$


with $\boldsymbol{\alpha}=\left(\alpha_{0}, \ldots, \alpha_{2 M}\right)^{T} \in \mathbb{R}^{2 M+1}$ for some fixed number $M \in N$. Furthermore, we set the variation $\mathbf{h}(\theta)=$ $\left\{(q(\theta) \cos \theta, q(\theta) \sin \theta)^{T}: \theta \in[0,2 \pi)\right\}$. From the representation (29), we have

$$
\begin{gathered}
\frac{\mathrm{d}}{\mathrm{d} s}=\frac{1}{\sqrt{r_{\alpha}^{2}(\theta)+\left[r_{\alpha}^{\prime}(\theta)\right]^{2}}} \frac{\mathrm{d}}{\mathrm{d} \theta}, \\
\mathbf{h} \cdot \mathbf{n}=\frac{q r}{\sqrt{r_{\alpha}^{2}+\left[r_{\alpha}^{\prime}\right]^{2}}} .
\end{gathered}
$$

4.2. Discrete Domain Derivative Equations. We define $U_{M}:=$ $\left\{\boldsymbol{\alpha} \in \mathbb{R}^{2 M+1}: \rho_{1} \leq r_{\alpha}(\theta) \leq \rho_{2}, \theta \in[0,2 \pi]\right\}, 0<\rho_{1}<\rho_{2}$. A simple application of Theorem 1 shows the following.

Theorem 2. For $\boldsymbol{\alpha} \in U_{M}$, the operator $F$ is differentiable with $\partial F_{i}(\alpha) / \partial \alpha_{j}=\partial_{n} u_{j}^{\prime}\left(x_{i}\right)$ for $i=1, \ldots, Q$ and $j=$ $0, \ldots, 2 M$. Here $u^{\prime} \in L^{2}\left(0, T ; H_{0}^{1}(\Omega)\right)$ is the solution of the initial boundary value problem

$$
\begin{gathered}
\frac{\partial u_{j}^{\prime}}{\partial t}+\mathbf{b} \cdot \nabla u_{j}^{\prime}-\operatorname{div}\left(a \nabla u_{j}^{\prime}\right)=0 \quad \mathbf{x} \in \Omega, t \in(0, T], \\
u_{j}^{\prime}=0 \quad \mathbf{x} \in \Gamma_{1}, t \in(0, T], \\
u_{j}^{\prime}=-k \frac{\partial u_{j}}{\partial n} \quad \mathbf{x} \in \Gamma_{2}, t \in(0, T], \\
u_{j}^{\prime}(\mathbf{x}, 0)=0, \quad \mathbf{x} \in \Omega,
\end{gathered}
$$

where

$$
k=-\frac{r_{\alpha}(\theta)}{\sqrt{r_{\alpha}^{\prime}(\theta)^{2}+r_{\alpha}(\theta)^{2}}} \begin{cases}\cos j \theta & j=0, \ldots, M \\ \sin (j-M) \theta & j=M+1, \ldots, 2 M\end{cases}
$$

for $\theta \in[0,2 \pi)$.

4.3. Regularized Gauss-Newton Algorithm. The iterative algorithm can be summarized as follows.

Step 1. Choose an initial boundary for $\Gamma_{2}$ and describe it by the parametric representations $\alpha^{0}$.

Step 2. Solve the advection-diffusion equations (1) by the finite element method.

Step 3. For a given $\alpha^{n}$, evaluate the Jacobian matrix of the mapping $F$ by solving the discrete domain derivative equations

$$
J\left(\alpha^{n+1}\right)=\left[\begin{array}{cccc}
\frac{\partial F_{1}\left(\alpha^{n}\right)}{\partial \alpha_{0}^{n}} & \frac{\partial F_{1}\left(\alpha^{n}\right)}{\partial \alpha_{1}^{n}} & \cdots & \frac{\partial F_{1}\left(\alpha^{n}\right)}{\partial \alpha_{2 M}^{n}} \\
\vdots & \vdots & \ddots & \vdots \\
\frac{\partial F_{Q}\left(\alpha^{n}\right)}{\partial \alpha_{0}^{n}} & \frac{\partial F_{Q}\left(\alpha^{n}\right)}{\partial \alpha_{1}^{n}} & \cdots & \frac{\partial F_{Q}\left(\alpha^{n}\right)}{\partial \alpha_{2 M}^{n}}
\end{array}\right]
$$

Step 4. Apply the regularized Gauss-Newton method to obtain the new approximation of boundary $\Gamma_{2}$,

$$
\begin{aligned}
\alpha^{n+1}= & \alpha^{n}-\left(J\left(\alpha^{n}\right)^{T} J\left(\alpha^{n}\right)\right)^{-1} J\left(\alpha^{n}\right) r\left(\alpha^{n}\right), \\
\text { where } r\left(\alpha^{n}\right)= & \left(F_{1}\left(\alpha^{n}\right)-P_{1}, \ldots, F_{Q}\left(\alpha^{n}\right)-P_{Q}\right)^{T} . \text { If } \\
& \sum_{i=1}^{Q}\left|F_{i}\left(\alpha^{n}\right)-P_{i}\right|+\mu\|\alpha\|^{2}<\varepsilon,
\end{aligned}
$$

where $\mu$ is a regularization parameter, then terminate, otherwise go back to Step 2.

4.4. Numerical Examples. We carry out the numerical examples to demonstrate the feasibility and validity of the proposed algorithm in Section 4.3.

In the following, we consider the shape reconstruction of the advection-diffusion process for the transport of a contaminant in two dimensions. We choose $D$ to be a rectangle $[-4,5] \times[-1.5,1.5]$ with the fixed boundary $\Gamma_{1}$, and the boundary $\Gamma_{2}$ of solid $S$ is to be recovered in our simulations. The velocity of water flow is imposed by $\mathbf{b}=$ $\left(0.25-y^{2}, 0\right)$, the diffusion coefficient is choose as $a=10^{-2}$, and the time interval is $[0,1]$.

We will reconstruct the shapes of solid $S$ with different boundary curves.

Case 1. An elliptic curve is defined by $x(t)=0.8 * \cos t, y=$ $0.6 * \sin t, t \in[0,2 \pi]$

Case 2. A cone-shaped curve is parametrized by $x(t)=0.7 *$ $\cos t+0.2 *(\cos 2 t-1), y(t)=0.5 * \sin t, t \in[0,2 \pi]$.

The dimension of the space $U_{M}$ is $2 M+1=25$, and the number of observation points is $Q=48$. We apply the finite element method to present the numerical implementation. Time discretization is effected using the backward Euler method and we assume that the time interval $[0,1]$ is divided into equal intervals of duration $\Delta t=$ 0.05 . Spatial discretization is effected using the continuous piecewise quadratic polynomials on a triangular mesh [15].

Figures 1 and 2 display the finite element mesh for two different target curves. Figures 3, 4, 5, and 6 demonstrate the comparison between the target shape and the iterated shape at different time. The solid line represents the exact boundary, and the dashed line gives the approximate boundary. The numerical results show that the iterative algorithm gives good reconstruction.

\section{Conclusions}

In this paper, we discuss the shape reconstruction problem governed by unsteady advection-diffusion equations. The differentiability of solution of the initial boundary value problem with respect to the boundary in the sense of the domain derivative is established, which is the theoretical 


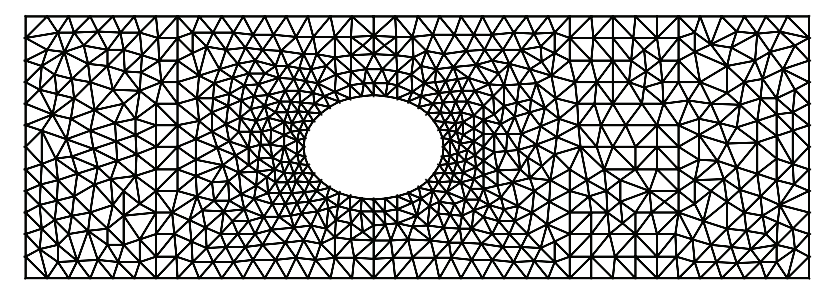

Figure 1: Case 1: finite element mesh for the target curve.

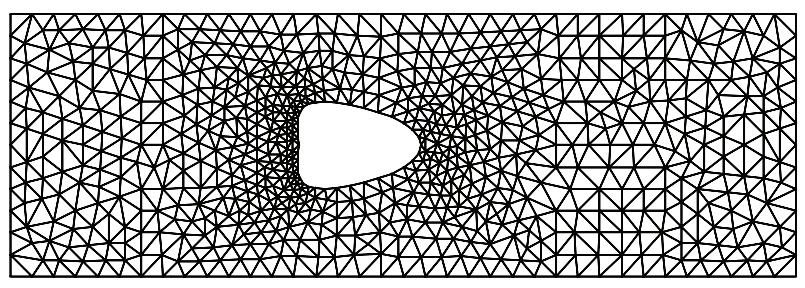

Figure 2: Case 2: finite element mesh for the target curve.

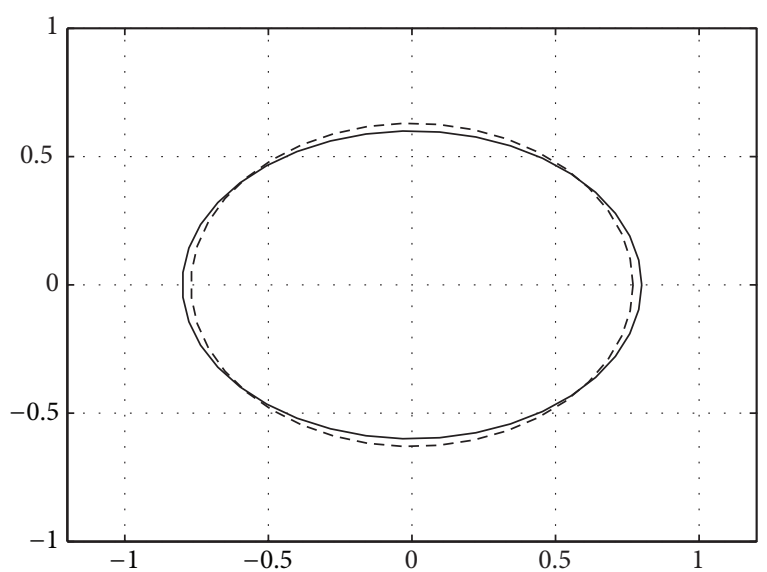

Figure 3: Case 1: $t=0.20$, reconstruction of an elliptic curve.

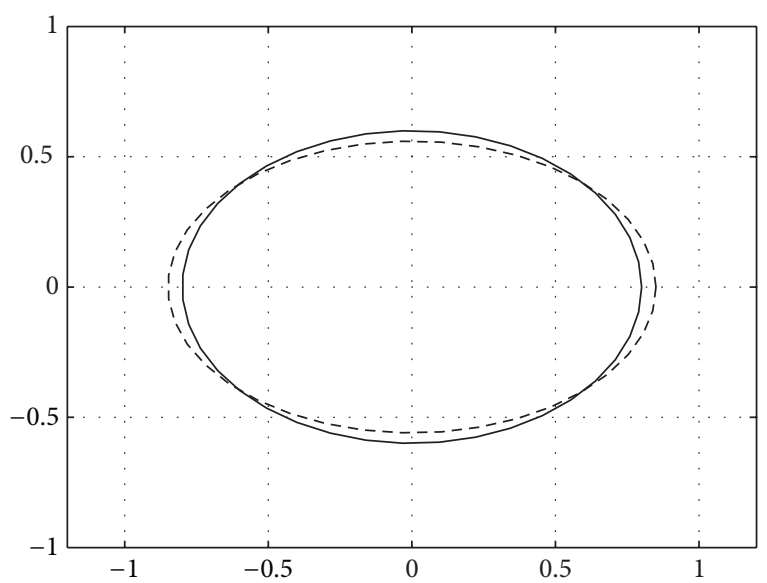

Figure 4: Case 1: $t=0.40$, reconstruction of an elliptic curve.

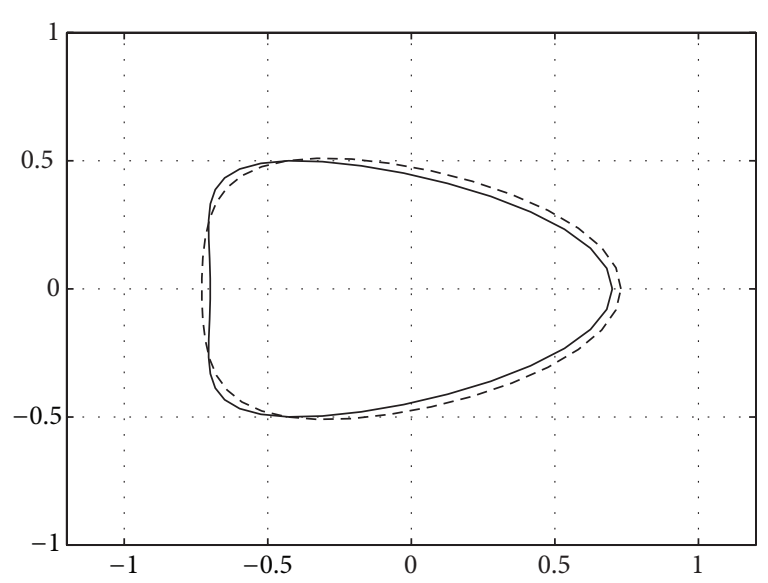

FIGURE 5: Case 2: $t=0.15$, reconstruction of a cone-shaped curve.

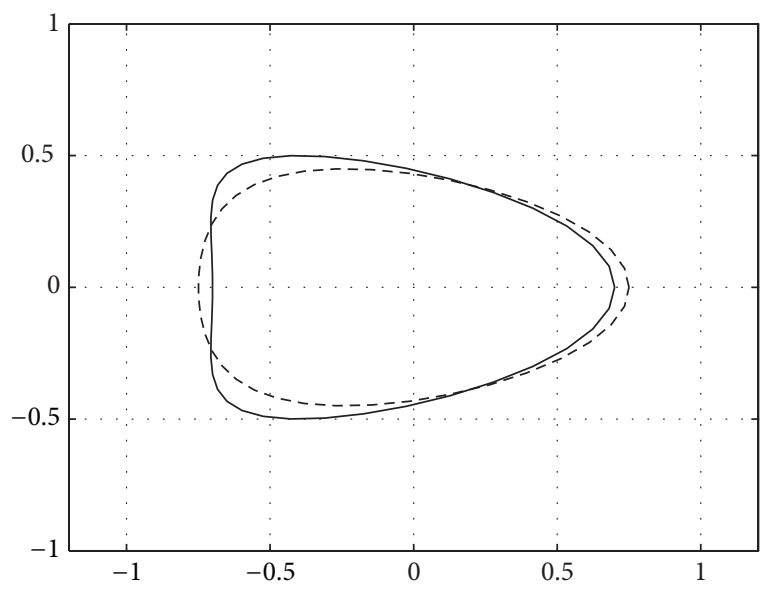

FIgure 6: Case 2: $t=0.30$, reconstruction of a cone-shaped curve.

foundation for the Newton method. A regularized GaussNewton scheme is effectively applied to the shape determination problem. Numerical experiments indicate the feasibility of the proposed method.

\section{Conflict of Interests}

The authors declare that there is no conflict of interests regarding the publication of this paper.

\section{Acknowledgments}

This work is supported by the National Natural Science Foundation of China (nos. 11371288, 11001216, and 11371289) and the Fundamental Research Funds for the Central Universities.

\section{References}

[1] F. Hettlich, "Fréchet derivatives in inverse obstacle scattering," Inverse Problems, vol. 11, no. 2, pp. 371-382, 1995. 
[2] F. Hettlich, "The Landweber iteration applied to inverse conductive scattering problems," Inverse Problems, vol. 14, no. 4, pp. 931-947, 1998.

[3] F. Hettlich and W. Rundell, "The determination of a discontinuity in a conductivity from a single boundary measurement," Inverse Problems, vol. 14, no. 1, pp. 67-82, 1998.

[4] R. Kress and W. Rundell, "Inverse scattering for shape and impedance," Inverse Problems, vol. 17, no. 4, pp. 1075-1085, 2001.

[5] R. Chapko, R. Kress, and J.-R. Yoon, "An inverse boundary value problem for the heat equation: the Neumann condition," Inverse Problems, vol. 15, no. 4, pp. 1033-1046, 1999.

[6] R. Chapko, R. Kress, and J.-R. Yoon, "On the numerical solution of an inverse boundary value problem for the heat equation," Inverse Problems, vol. 14, no. 4, pp. 853-867, 1998.

[7] H. Harbrecht and J. Tausch, "On the numerical solution of a shape optimization problem for the heat equation," SIAM Journal on Scientific Computing, vol. 35, no. 1, pp. A104-A121, 2013.

[8] H. Harbrecht and J. Tausch, "An efficient numerical method for a shape-identification problem arising from the heat equation," Inverse Problems, vol. 27, no. 6, article 065013, 2011.

[9] W.-J. Yan and Y.-C. Ma, "The application of domain derivative for heat conduction with mixed condition in shape reconstruction," Applied Mathematics and Computation, vol. 181, no. 2, pp. 894-902, 2006.

[10] W.-J. Yan and Y.-C. Ma, "Shape reconstruction of an inverse Stokes problem," Journal of Computational and Applied Mathematics, vol. 216, no. 2, pp. 554-562, 2008.

[11] A. Quarteroni and A. Valli, Numerical Approximation of Partial Differential Equations, vol. 23 of Springer Series in Computational Mathematics, Springer, Berlin, Germany, 1994.

[12] R. A. Adams and J. J. F. Fournier, Sobolev Spaces, vol. 140 of Pure and Applied Mathematics, Elsevier/Academic Press, Amsterdam, The Netherlands, 2nd edition, 2003.

[13] O. Pironneau, Optimal Shape Design for Elliptic Systems, Springer Series in Computational Physics, Springer, Berlin, Germany, 1984.

[14] M. C. Delfour and J.-P. Zolésio, Shapes and Geometries: Analysis, Differential Calculus and Optimization, Advance in Design and Control, Springer, Berlin, Germany, 2002.

[15] V. Isakov, Inverse Problem for Partial Differential Equations, Spring, New York, NY, USA, 1998. 


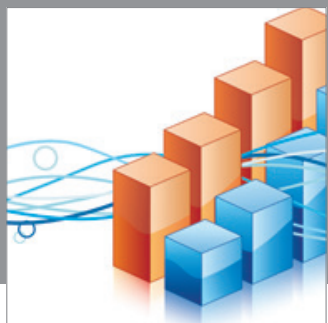

Advances in

Operations Research

mansans

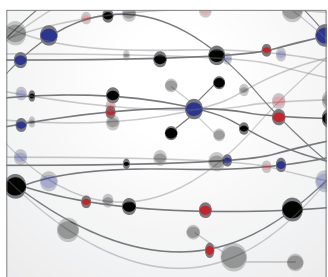

The Scientific World Journal
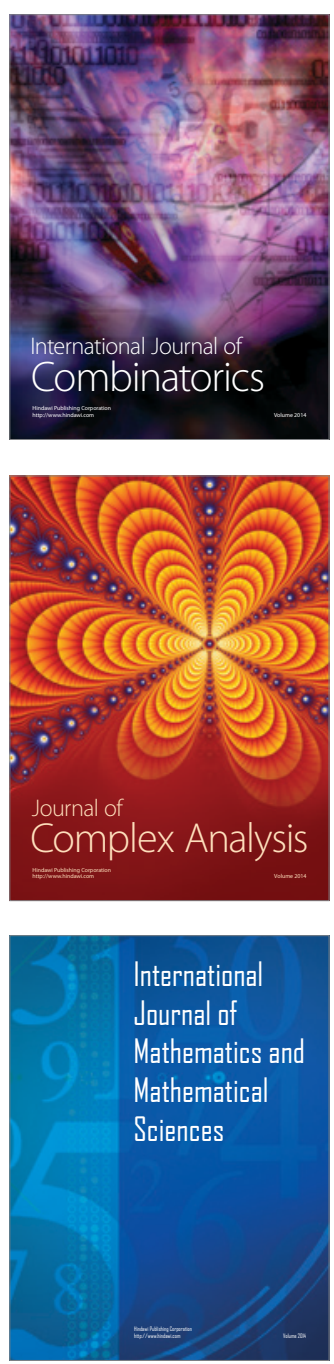
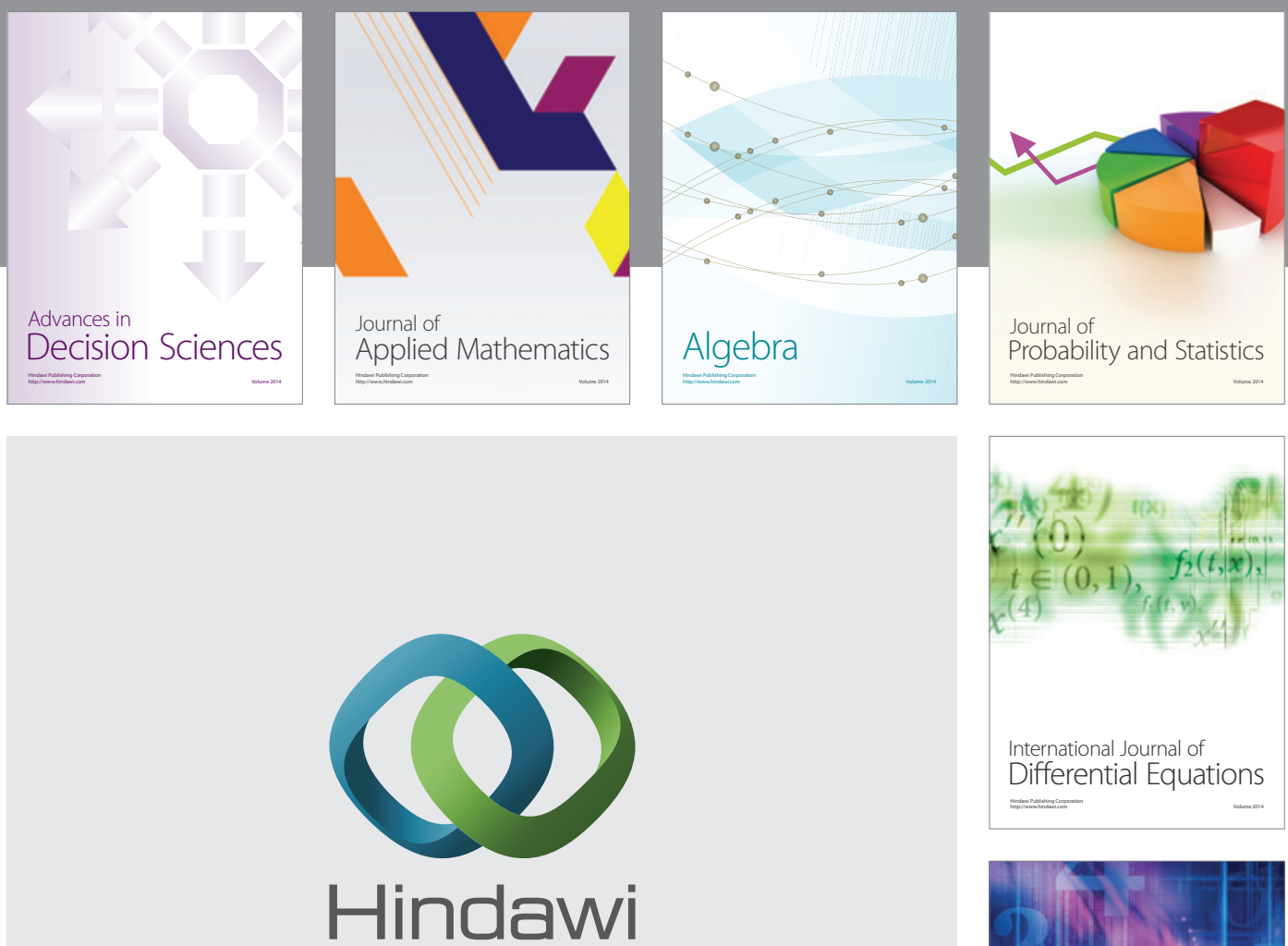

Submit your manuscripts at http://www.hindawi.com
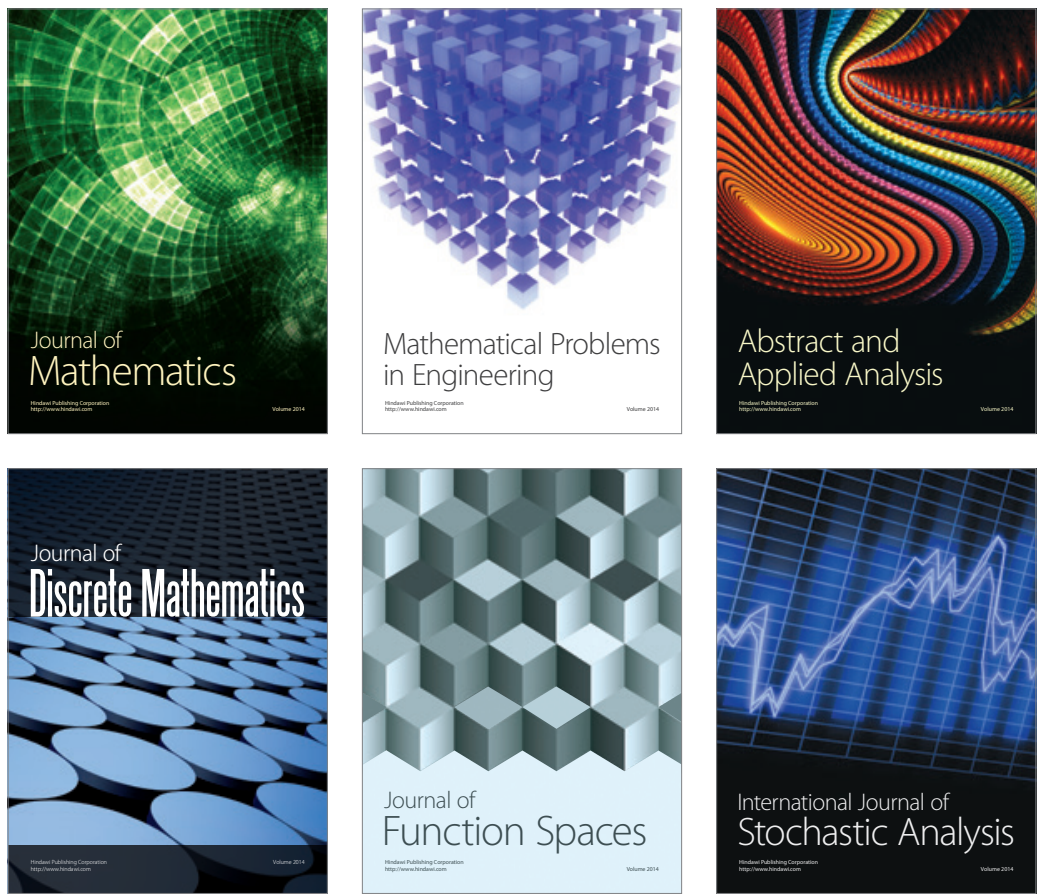

Journal of

Function Spaces

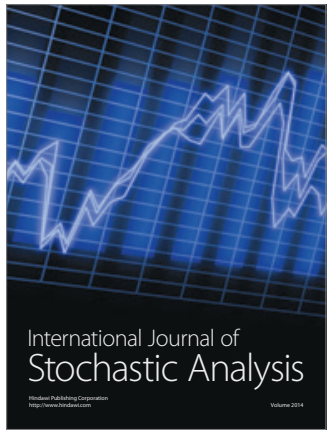

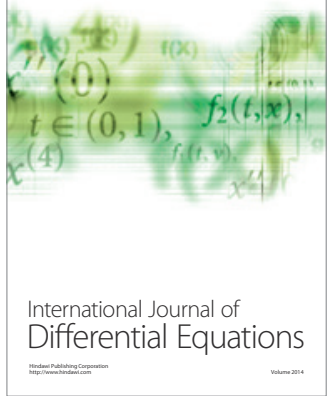
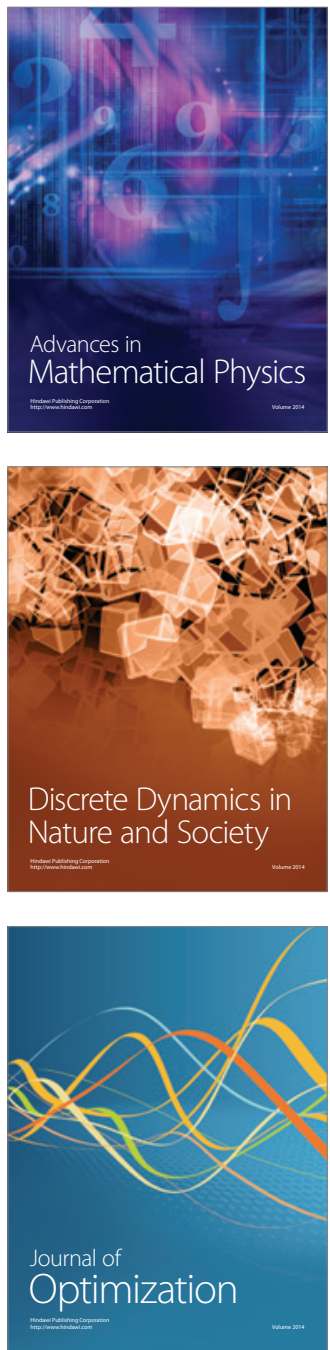